\title{
Pauli principle and the Monte Carlo method for charge transport in graphene
}

\author{
Marco Coco $\odot,{ }^{1, *}$ Paolo Bordone $\odot,{ }^{2, \dagger}$ Lucio Demeio $\odot,{ }^{1, \$}$ and Vittorio Romano $\oplus^{3, \S}$ \\ ${ }^{1}$ Dipartimento di Ingegneria Industriale e Scienze Matematiche, Università Politecnica delle Marche, via Brecce Bianche 12, \\ 60131 Ancona, Italy \\ ${ }^{2}$ Dipartimento di Scienze Fisiche, Informatiche e Matematiche, Università di Modena e Reggio Emilia, via Campi 213/A, 41125 Modena, Italy \\ ${ }^{3}$ Dipartimento di Matematica e Informatica, Università degli Studi di Catania, viale Andrea Doria 6, 95125 Catania, Italy
}

(Received 5 July 2021; revised 12 October 2021; accepted 18 October 2021; published 8 November 2021)

\begin{abstract}
An attempt to include the Pauli principle in the Monte Carlo method by also acting on the free-flight step and not only at the end of each collision is investigated. The charge transport in suspended monolayer graphene is considered as a test case. The results are compared with those obtained with the standard ensemble Monte Carlo technique and with the updated direct simulation Monte Carlo algorithm which is able to correctly handle with Pauli's principle. The physical aspects of the investigated approach are analyzed as well.
\end{abstract}

DOI: 10.1103/PhysRevB.104.205410

\section{INTRODUCTION}

The Monte Carlo approach is nowadays widely used in simulations of charge transport in semiconductor devices. Most of the methods employed in this field are based on the ensemble Monte Carlo (EMC) procedure of Lebwohl and Price [1] and on the approach developed by Bosi, Jacoboni, and Reggiani [2,3]. When the simulations involve degenerate materials, the inclusion of the Pauli principle becomes essential; to this aim, Lugli and Ferry [4] improved the EMC method and included the Pauli principle by means of a rejection technique at the end of each scattering process. Unfortunately, with this procedure the charge distribution can exceed the maximum value of 1 , leading to unphysical results. Some attempts were made in the literature to overcome this difficulty: in $[5,6]$ ad hoc scattering out terms to force the distribution function to be smaller than 1 were introduced; in $[7,8]$ some approximations of the distribution of the final states were used. These efforts improved the simulation results but did not lead to the correct reconstruction of the distribution function, which is of fundamental importance since it enters the collisional terms of the Boltzmann equation and influences the determination of the scattering probabilities.

An important issue which we address in this paper arises when degenerate materials are considered. In this case, there is an ongoing debate in the literature about whether the Pauli principle should be applied to the free-flight step or not. This is an important theoretical question for computational purposes as well. An alternative procedure was proposed in [9] in which the rejection technique was adopted not only at the end of each scattering event but also at the end of each free flight. To the best of our knowledge, this method, despite being cited several times in the literature (see, for

\footnotetext{
*Corresponding author: m.coco@univpm.it

†paolo.bordone@unimore.it

†1.demeio@ univpm.it

§romano@dmi.unict.it
}

example, $[5,6,8,10,11])$, has never been used. In the literature an analysis of this approach is missing (it received only a brief comment in [5]); it is the aim of this work to fill this gap and to present a coherent discussion on the inclusion of the Pauli principle in a Monte Carlo procedure. We do so by comparing the numerical results for a suspended monolayer graphene obtained with the Monte Carlo Method presented in [9] with those obtained using the standard EMC in [4] and the updated direct simulation Monte Carlo (DSMC) in [12], which are by now well established in the semiconductor field and have been cross validated with deterministic solutions, for example, those based on the discontinuous Galerkin method [12-19] or on weighted essentially non-oscillatory (WENO) schemes [20].

The updated DSMC scheme in [12] is able to correctly include the Pauli principle; the streaming term of the Boltzmann equation is treated deterministically by means of a splitting procedure, resulting in a rigid translation of the distribution function as a whole. The scattering events are then simulated, and the rejection technique is applied at the end of each collision.

The results obtained using the EMC and the DSMC were already studied in [12] and allow us to quantify the correctness of the method proposed in [9]. In addition, graphene is a material whose peculiar energy bands make the degeneracy effects relevant, thus representing a useful choice as a test case.

The relationship between the results of a Monte Carlo simulation and the solution of the Boltzmann equation is still a matter of debate in the literature. Almost all the Monte Carlo approaches for studying charge transport in semiconductor materials are based on the so-called direct simulation which allows us to follow the single-particle dynamics (and later to calculate the mean quantities). The direct simulation approach is derived from Bird's algorithm in gas dynamics [21,22], for which a convergence proof to the solution of the Boltzmann equation is given in [23]. The direct simulation approach can be interpreted as only one possible application of the Monte Carlo technique corresponding to a particular choice of the 
scattering probabilities, which are, in our case, the physical scattering rates. When the probabilities of the events are defined in a general arbitrary way, the Monte Carlo method, called in this case weighted Monte Carlo, can be interpreted as a formal solution of the Boltzmann equation. For further details we refer the interested reader to [24] and references therein.

This paper is organized as follows. In Sec. II the semiclassical mathematical model for spatially homogeneous graphene is presented, and the simulation procedures are introduced; in Sec. III a preliminary analysis of the numerical accuracy of the standard and updated Monte Carlo methods is carried out; in Sec. IV we present and discuss the results of our simulations. Section V contains our conclusions.

\section{THE MATHEMATICAL MODEL AND THE MONTE CARLO TECHNIQUES}

The carrier population in graphene is made up of four components: the electrons of the valence and conduction bands, which can occupy the states around either of the Dirac points, $K$ and $K^{\prime}$, of each band. In our simulations, we consider a homogeneous graphene strip of infinite extension in the direction transverse to the applied electric field and only the electrons of the conduction band belonging to the valley around the $K$ point because we consider the two valleys to be equivalent. We recall that the graphene Brillouin zone $\mathcal{B}$ has a hexagonal shape, and we shall choose the reference frame of the $\mathbf{k}$ space so that the origin coincides with the $K$ point. The electric field is directed along the $x$ axis.

With good approximation [25], the dispersion relation for the band energy $\varepsilon$ around the equivalent Dirac points is given by

$$
\varepsilon=\hbar v_{F}\left|\mathbf{k}-\mathbf{k}_{\ell}\right|,
$$

where $v_{F}$ is the Fermi velocity and $\mathbf{k}_{\ell}$ is the position of the Dirac point $\ell$. We will use Eq. (1) as the dispersion relation because for the electric field strengths usually considered in applications the charge transport involves almost exclusively the electrons around the $K$ and $K^{\prime}$ points.

Under these conditions and using the semiclassical approximation, the Boltzmann equation for the charge carriers is

$$
\frac{\partial f(t, \mathbf{k})}{\partial t}-\frac{e}{\hbar} E \frac{\partial f(t, \mathbf{k})}{\partial k_{x}}=\left.\frac{d f}{d t}(t, \mathbf{k})\right|_{e-\mathrm{ph}},
$$

where $f(t, \mathbf{k})$ is the electron distribution function of the charge carriers at time $t, \mathbf{k}=\left(k_{x}, k_{y}\right)$ is the wave vector. The right-hand side of Eq. (2) is the collision operator which describes the interactions of the carriers with the phonons.

The appropriate initial condition for Eq. (2) in the degenerate case is the Fermi-Dirac distribution

$$
f(0, \mathbf{k})=f_{F D}(\mathbf{k}) \equiv \frac{1}{1+\exp \left(\frac{\varepsilon(\mathbf{k})-\varepsilon_{F}}{k_{B} T}\right)},
$$

where $\varepsilon_{F}$ is the Fermi level and $T$ is the room temperature, related to the charge distribution by means of

$$
\rho(0)=\frac{2}{(2 \pi)^{2}} \int f(0, \mathbf{k}) d^{2} \mathbf{k},
$$

where only the spin degeneracy is considered. The Fermi level will be chosen to be high enough to produce a strong degeneracy, according to the situation we want to investigate. This is equivalent to introducing a high $n$ doping in a traditional semiconductor.

The electron mean energy and velocity are defined as

$$
\begin{aligned}
& \mathcal{E}(t)=\frac{1}{\rho(t)} \frac{2}{(2 \pi)^{2}} \int \varepsilon(t, \mathbf{k}) f(t, \mathbf{k}) d^{2} \mathbf{k}, \\
& \mathbf{V}(t)=\frac{1}{\rho(t)} \frac{2}{(2 \pi)^{2}} \int \mathbf{v}(t, \mathbf{k}) f(t, \mathbf{k}) d^{2} \mathbf{k},
\end{aligned}
$$

where $\varepsilon(t, \mathbf{k})$ and $\mathbf{v}(t, \mathbf{k})$ are the particle energy and velocity, respectively, and $\rho(t)$ is the time-dependent electron density,

$$
\rho(t)=\frac{2}{(2 \pi)^{2}} \int f(t, \mathbf{k}) d^{2} \mathbf{k} .
$$

The collision operator represents the interactions of the electrons with acoustic, optical, and $K$ phonons. Acoustic phonon scattering is intravalley and intraband and can be longitudinal (LA) or transverse (TA). Optical phonon scattering is intravalley and can be longitudinal (LO) and transverse (TO); it can be intraband, leaving the electrons in the same band, or interband, pushing the electrons from the initial band toward another one. Scattering with $K$ phonons pushes electrons from a valley to a nearby one (intervalley scattering). For the optical and $K$ phonons we will assume the Einstein approximation, $\hbar \omega_{A}=$ const, $A=\mathrm{LO}, \mathrm{TO}, K$, with $\omega_{A}$ being the $A$ th phonon frequency. The $K$ phonons are not an actual physical phonon branch; their name is due to the fact that their wave vectors are close to the $K$ or $K^{\prime}$ point [25]. They belong to the optical branches and induce intervalley scatterings. This justifies the use of the Einstein approximation for them. For the in-plane acoustic phonons the Debye approximation will be adopted, $\hbar \omega_{A}=\hbar v_{A}|\mathbf{q}|, A=\mathrm{LA}$, TA, with $\mathbf{q} \in \mathcal{B}$ being the phonon wave vector, and in this case for the analytical calculations the Brillouin zone can be consistently extended to $\mathbb{R}^{2}$. The out-of-plane $Z$ phonons are not considered because they do not interact with the electrons; they are important when phonon transport and thermal effects are taken into account [26-29].

The general form of the collision term can be written as $[20,25]$

$$
\begin{aligned}
\left.\frac{d f}{d t}(t, \mathbf{k})\right|_{e-\mathrm{ph}}= & \int_{\mathcal{B}} S\left(\mathbf{k}^{\prime}, \mathbf{k}\right) f\left(t, \mathbf{k}^{\prime}\right)[1-f(t, \mathbf{k})] d \mathbf{k}^{\prime} \\
& -\int_{\mathcal{B}} S\left(\mathbf{k}, \mathbf{k}^{\prime}\right) f(t, \mathbf{k})\left[1-f\left(t, \mathbf{k}^{\prime}\right)\right] d \mathbf{k}^{\prime},
\end{aligned}
$$

where $S\left(\mathbf{k}^{\prime}, \mathbf{k}\right)$ is the total transition rate and is given by the sum over several types of scatterings whose details are reported in the Appendix,

$$
\begin{aligned}
S\left(\mathbf{k}^{\prime}, \mathbf{k}\right)= & \sum_{A}\left|G^{(A)}\left(\mathbf{k}^{\prime}, \mathbf{k}\right)\right|^{2}\left\{\left(g_{A}^{-}+1\right) \delta\left[\varepsilon(\mathbf{k})-\varepsilon\left(\mathbf{k}^{\prime}\right)+\hbar \omega_{A}\right]\right. \\
& \left.+g_{A}^{+} \delta\left[\varepsilon(\mathbf{k})-\varepsilon\left(\mathbf{k}^{\prime}\right)-\hbar \omega_{A}\right]\right\},
\end{aligned}
$$

where the index $A$ runs over the phonon modes: longitudinalacoustical (LA) and -optical (LO), transverse-acoustical (TA) and -optical (TO), and $K$ phonons. $\left|G^{(A)}\left(\mathbf{k}^{\prime}, \mathbf{k}\right)\right|^{2}$ are the 
electron-phonon coupling matrix elements, which describe the interaction mechanism of an electron with the $A$ th phonon, from the state of wave vector $\mathbf{k}^{\prime}$ to the state of wave vector $\mathbf{k}$. The symbol $\delta$ denotes the Dirac delta function, and $g_{A}(\mathbf{q})$ is the phonon distribution for the $A$-type phonons. In (9), $g_{A}^{ \pm}=g_{A}\left(\mathbf{q}^{ \pm}\right)$, where $\mathbf{q}^{ \pm}= \pm\left(\mathbf{k}^{\prime}-\mathbf{k}\right)$, stemming from the momentum conservation.

In the Monte Carlo procedure, the scattering rate of each type of scattering plays a fundamental role; the scattering rate $\Gamma_{A}$ of the $A$ th type of scattering is defined as

$$
\Gamma_{A}(\mathbf{k})=\int_{\mathcal{B}} S_{A}\left(\mathbf{k}, \mathbf{k}^{\prime}\right) d \mathbf{k}^{\prime} .
$$

The expressions for all types of scattering mechanisms are reported in the Appendix.

In this work, we compare the results for charge transport in graphene obtained with three different Monte Carlo procedures: the standard ensemble MC procedure, an updated ensemble MC procedure, and the free-flight-based one.

In general, in a Monte Carlo simulation, the motion of each particle is given by the solution of the semiclassical equation of motion $\hbar \dot{\mathbf{k}}=-e \mathbf{E}$, followed by a collisional event after a time $\Delta t$. The total scattering rate is given by

$$
\tilde{\Gamma}_{\mathrm{tot}}=\Gamma_{\mathrm{LA}}+\Gamma_{\mathrm{TA}}+\Gamma_{\mathrm{LO}}+\Gamma_{\mathrm{TO}}+\Gamma_{K},
$$

and it varies with the energy (see the Appendix for details about the scatterings). A scattering rate $\Gamma_{s s}$ due to fictitious collision events, which are called self-scatterings and do not change the particle state, is also introduced, and the new constant total scattering rate is defined as $\Gamma_{\text {tot }}=\tilde{\Gamma}_{\text {tot }}+\Gamma_{\text {ss }}$. $\Delta t$ is then calculated as the ratio (see, for example, [30])

$$
\Delta t=-\frac{\ln \eta}{\Gamma_{\text {tot }}},
$$

where $\eta$ is a random number uniformly distributed in $[0,1]$. The free flight is always present, and it is always interrupted by a scattering event. The free-flight time is of the order of a few hundred femtoseconds [25]. In general, the value of $\Gamma_{\text {tot }}$ is determined as $\alpha \Gamma_{\max }$, with $\Gamma_{\max }=\max \left(\Gamma_{\mathrm{LA}}+\Gamma_{\mathrm{TA}}+\right.$ $\Gamma_{\mathrm{LO}}+\Gamma_{\mathrm{TO}}+\Gamma_{K}$ ), where $\alpha>1$ is a tuning parameter. Since the previous scattering rates can differ even by two orders of magnitude, using the same $\Gamma_{\text {tot }}$ for each time step leads to a very large number of self-scatterings, making the computational cost considerably high. Therefore, in our simulations, we use a variable $\Gamma_{\text {tot }}$ that depends on the state of the particle at the current time $t$ :

$$
\begin{aligned}
\Gamma_{\mathrm{tot}}= & \alpha\left[\Gamma_{\mathrm{LA}}(\varepsilon(t))+\Gamma_{\mathrm{TA}}(\varepsilon(t))+\Gamma_{\mathrm{LO}}(\varepsilon(t))\right. \\
& \left.+\Gamma_{\mathrm{TO}}(\varepsilon(t))+\Gamma_{K}(\varepsilon(t))\right],
\end{aligned}
$$

with $\alpha=1.1$.

In our simulations, the following three Monte Carlo procedures will be used: (i) standard ensemble Monte Carlo (SEMC) [4], (ii) the updated, or "new," ensemble Monte Carlo (NEMC) [12], and (iii) the free-flight-based Monte Carlo (FFMC) [9].

(i) SEMC. After each collision, the new wave vector $\mathbf{k}^{\prime}$ is determined, and if the final state is available, the initial state $\mathbf{k}$ is updated. The availability of the final states has to respect the Pauli principle, and this is checked by means of a rejection procedure: a random number $\zeta$, uniformly distributed in $[0,1]$, is generated, and the final state $\mathbf{k}^{\prime}$ is available if the condition $\zeta<1-f\left(\mathbf{k}^{\prime}\right)$ holds. This scheme is repeated for each particle. The main steps of the SEMC procedure for each time window $\left[t_{n}, t_{n+1}\right]$ are as follows:

(a) Choose the free flight duration for each particle according to Eq. (12).

(b) Solve the semiclassical equation of motion during the free flight in the $\mathbf{k}$ space for each particle

$$
\frac{d(\hbar \mathbf{k})}{d t}=-e \mathbf{E} .
$$

(c) Choose the type of scattering and calculate the energy and the momentum of the particle after the collision with final state $\mathbf{k}^{\prime}$.

(d) Use the rejection technique to check the availability of the final state $\mathbf{k}^{\prime}$.

(e) Update the values of the energy, momentum of the particle, and the distribution function $f$ if the final state is available; otherwise, the scattering is rejected, and it does not happen.

(f) Generate a new free-flight time and repeat the procedure until $t_{n+1}$ is reached.

(g) At $t=t_{n+1}$ evaluate and record the mean energy and the velocity.

(ii) NEMC. This method consists of two main steps. First, the distribution function is translated as a whole according to the semiclassical equation of motion, and all the particles experience the same free flight; steps 1 and 2 of the SEMC are replaced by the solution of Eq. (2) by setting the right-hand side equal to zero: $f\left(t+\Delta t, \mathbf{k}^{\prime}\right)=f\left(t, \mathbf{k}+\frac{e}{\hbar} \mathbf{E} \Delta t\right)$. Then, for each particle a sequence of collisional events is simulated. The final state after each scattering mechanism is checked by using the rejection technique described above.

(iii) FFMC. In this method, the rejection technique to check the availability of the final states is used not only at the end of each collision but also at the end of each free flight; if the state reached after the free flight governed by the semiclassical equation of motion is not accepted, the particle goes back, and nothing happens. The algorithm is similar to the SEMC, but step 4 is used also after step 2 .

\section{ACCURACY OF THE METHODS}

In this section a preliminary analysis of the numerical accuracy of reference methods SEMC and NEMC is carried out.

We discretize the wave-vector space by means of a uniform square grid $\left[-k_{x, \max }, k_{x, \max }\right] \times\left[-k_{y, \max }, k_{y, \max }\right]$, with $k_{x, \text { max }}=$ $k_{y, \max }=24 \mathrm{~nm}^{-1}$ and $642 \times 642$ cells. For each cell $C_{\alpha}$, we consider the cell center with wave vector $\mathbf{k}_{\alpha}=\left(k_{i}, k_{j}\right)$, with $i$ and $j$ being the indices of the cell centers along the $x$ and $y$ directions, respectively. Let $n_{P}$ be the initial number of simulated particles. The particles are allocated in each cell according to the following Fermi-Dirac distribution $f_{i j}$, calculated in each cell center:

$$
f_{i j}\left(0, \mathbf{k}_{\alpha}\right)=\frac{1}{1+\exp \left(\frac{\varepsilon_{i j}\left(\mathbf{k}_{\alpha}\right)-\varepsilon_{F}}{k_{B} T}\right)},
$$


TABLE I. Physical parameters for the scattering rates.

\begin{tabular}{ll}
\hline \hline Symbol & Value \\
\hline$\sigma_{m}$ & $7.6 \times 10^{-8} \mathrm{~g} / \mathrm{cm}^{2}$ \\
$v_{F}$ & $10^{6} \mathrm{~m} / \mathrm{s}$ \\
$v_{\mathrm{LA}}$ & $2.13 \times 10^{4} \mathrm{~m} / \mathrm{s}$ \\
$v_{\mathrm{TA}}$ & $1.36 \times 10^{4} \mathrm{~m} / \mathrm{s}$ \\
$D_{a c}$ & $6.8 \mathrm{eV}$ \\
$\hbar \omega_{\mathrm{LO}}$ & $164.6 \mathrm{meV}$ \\
$\hbar \omega_{\mathrm{TO}}$ & $164.6 \mathrm{meV}$ \\
$D_{O}$ & $10^{9} \mathrm{eV} / \mathrm{cm}$ \\
$\hbar \omega_{K}$ & $124 \mathrm{meV}$ \\
$D_{K}$ & $3.5 \times 10^{8} \mathrm{eV} / \mathrm{cm}$ \\
\hline \hline
\end{tabular}

where $\varepsilon_{F}$ is the Fermi level, $T$ is the room temperature, and $\varepsilon_{i j}$ is the energy associated with each cell center using the dispersion relation

$$
\varepsilon_{i j}=\hbar v_{F}\left|\mathbf{k}_{\alpha}\right|=\hbar v_{F}\left|\left(k_{i}, k_{j}\right)\right| .
$$

In each cell, we allocate $n_{i j}=f_{i j} \times n_{P}$ particles; the $n_{i j}$ particles of the $C_{\alpha}$ cell have the wave vector of the corresponding cell center $\mathbf{k}_{\alpha}=\left(k_{i}, k_{j}\right)$ and energy $\varepsilon_{i j}$. We approximate each number of particles $n_{i j}$ with the nearest integer $\tilde{n}_{i j}$. So the number of particles in each cell of wave vector $\left(k_{i}, k_{j}\right)$ is $\tilde{n}_{i j}$, and the actual total number of simulated particles is

$$
\tilde{n}_{P}=\sum_{i, j} \tilde{n}_{i, j}
$$

During the simulation the distribution $\tilde{n}_{i, j}$ is updated as $\tilde{n}_{i, j}=\tilde{n}_{i, j} \pm 1$ if a charged particle reaches or leaves the state $(i, j)$, respectively. Eventually, by considering the maximum occupation number $M=\max _{i, j} \tilde{n}_{i, j}$, we define the numerical distribution function $\tilde{f}_{i, j}$ as

$$
\tilde{f}_{i, j}=\frac{\tilde{n}_{i, j}}{M} .
$$

For example, for $n_{P}=10^{4}$ particles we have $M=22$.

We compare the results for the numerical reconstruction of the distribution function of both the SEMC and NEMC approaches for several different values of the particle number, $n_{P}=(0.25,0.5,1,2,4,8,16,32) \times 10^{4}$. All the results reported here are obtained with an electric field $E$ equal to 20 $\mathrm{kV} / \mathrm{cm}$ and Fermi level $\varepsilon_{F}=0.6 \mathrm{eV}$. The physical parameters proposed in [31,32] and reported in Table I are adopted.

We evaluate the numerical distribution function $\tilde{f}_{i, j}$ at $t=5 \mathrm{ps}$ for the SEMC and NEMC methods for the different values of $n_{P}$. The results are shown and compared in Fig. 1. It can be noted that the Pauli principle is always fulfilled in the NEMC case for each value of the particle number $n_{P}$, while the numerical distribution function obtained with the SEMC approach is compatible with the Pauli principle only for $n_{P} \geqslant 8 \times 10^{4}$. In both cases, the maximum value of $\tilde{f}$ decreases as the number of particles increases due to the higher numerical accuracy. The right panels of Fig. 1 show how the grid is rigidly moved in the $x$-axis direction together with the whole distribution function, consistent with the semiLagrangian approach of the NEMC.

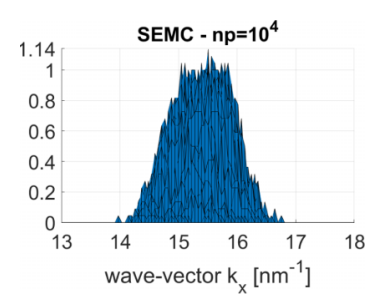

(a)

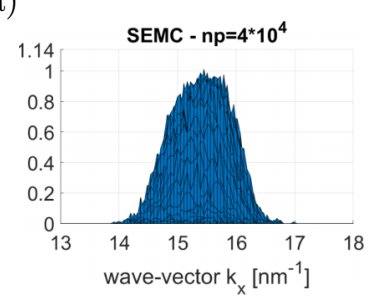

(b)

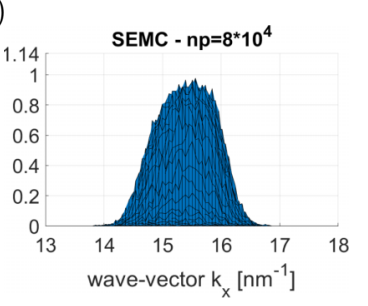

(c)

(d)

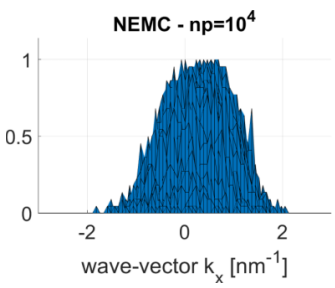

(e)
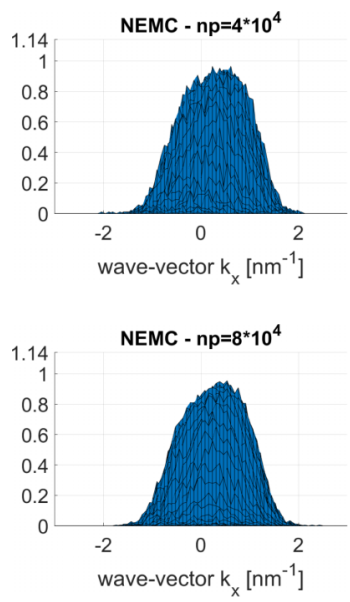

(f)

FIG. 1. View of the particle distribution along the $x$ axis for the different values of the particle number $n_{P}$.

In Table II we report the one-norm and two-norm of the difference $\Delta f_{1,2}=f_{2}-f_{1}$, where $f_{1}$ and $f_{2}$ are calculated with particle numbers $n_{1}$ and $n_{2}$. The results suggest that the NEMC method has higher numerical accuracy with a lower number of particles and that the NEMC and SEMC approaches reach a comparable numerical accuracy only with a very large number of particles, which makes SEMC computationally much more expensive than NEMC.

\section{SIMULATION RESULTS}

In this section, the results of the three Monte Carlo approaches to include the Pauli principle presented in Sec. II are shown, compared, and discussed.

In the simulations, $n_{P}=10^{4}$ (super)particles are used, and the time step is set equal to $\Delta t=2.5 \mathrm{fs}$; the wave-vector grid and the physical parameters are the same as in Sec. III.

TABLE II. The $(1,2)$-norm of the distribution function in the NEMC and SEMC cases for different numbers of particles.

\begin{tabular}{lcccc}
\hline \hline & NEMC \\
$n_{1}, n_{2}$ & $\begin{array}{c}\text { SEMC } \\
\left\|\Delta f_{1,2}\right\|_{1}\end{array}$ & $\begin{array}{c}\text { NEMC } \\
\left\|f_{1,2}\right\|_{1}\end{array}$ & $\begin{array}{c}\text { SEMC } \\
\left\|\Delta f_{1,2}\right\|_{2}\end{array}$ & $\left\|\Delta f_{1,2}\right\|_{2}$ \\
\hline$(0.25,0.5) \times 10^{4}$ & 7.7455 & 9.4727 & 2.3787 & 2.9031 \\
$(0.5,1) \times 10^{4}$ & 5.5909 & 6.2273 & 1.6949 & 1.9403 \\
$(1,2) \times 10^{4}$ & 4.2045 & 4.7955 & 1.2717 & 1.5014 \\
$(2,4) \times 10^{4}$ & 2.9378 & 3.9574 & 0.8351 & 0.9956 \\
$(4,8) \times 10^{4}$ & 2.1665 & 2.4407 & 0.5773 & 0.6817 \\
$(8,16) \times 10^{4}$ & 1.4425 & 1.6883 & 0.4099 & 0.5012 \\
$(16,32) \times 10^{4}$ & 1.0127 & 1.1548 & 0.3020 & 0.3427 \\
\hline \hline
\end{tabular}




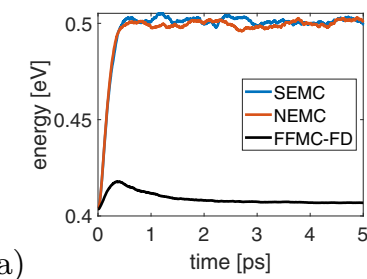

(b)

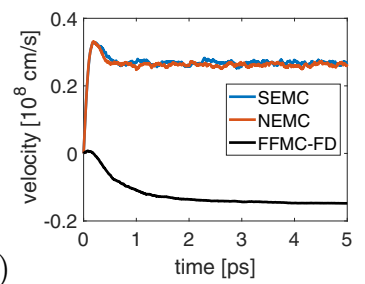

FIG. 2. (a) Mean energy and (b) velocity for the SEMC, NEMC, and FFMC procedures, with $\varepsilon_{F}=0.6 \mathrm{eV}$ and $E=20 \mathrm{kV} / \mathrm{cm}$.

In the previous section it was shown that the NEMC is able to properly take into account the Pauli principle even for a low number of particles, in contrast to SEMC, thanks to the higher numerical accuracy. Regarding the mean values, in Fig. 2 the mean energy and velocity for both schemes are reported; the results of the NEMC and SEMC methods are in good agreement (for further details see [12], where a cross validation with the deterministic results obtained by means of the discontinuous Galerkin method was analyzed as well).

In Fig. 2 the average energy and velocity obtained with the FFMC procedure are shown and compared with those obtained with SEMC and NEMC. The energy remains almost constant, and the velocity, after an initial negligible positive peak, reaches a negative value equal to $-0.14 \times 10^{8} \mathrm{~cm} / \mathrm{s}$. The behavior given by the FFMC approach is certainly unphysical because it is opposite to the one induced by the electric field. In [9], the unphysical results are explained as a result of the initial Fermi-Dirac condition, which leads to almost all the final states being unavailable.

The slight variation in the energy and in the absolute value of the velocity observed with the FFMC in Fig. 2 is compatible with an overestimation of the Pauli principle, which freezes the charge dynamics.

In the following sections, we discuss the main results, in particular for the charge distribution and the mean energy and velocity, when both an initial Fermi-Dirac distribution and a Maxwell-Boltzmann distribution are considered.

\section{A. Initial Maxwell-Boltzmann distribution}

To overcome the difficulty related to the frozen dynamics due to the Fermi-Dirac distribution, Tadyszak et al. [9] proposed to use a high-temperature Maxwell-Boltzmann distribution as the initial condition in place of the Fermi-Dirac one; the distribution temperature was heuristically set equal to $80 T$, with $T$ being the room temperature, for the silicon case. Along these lines, we introduce the following MaxwellBoltzmann distribution:

$$
f(0, \mathbf{k})=f_{M B}(\mathbf{k}) \equiv \exp \left(-\frac{\varepsilon-\mu}{k_{B} T^{*}}\right),
$$

where the free parameters $\mu$ and $T^{*}$ are the electrochemical potential and the temperature, respectively. They can be determined by setting equal the charge densities calculated with $f_{F D}$ and $f_{M B}$ : one of the two parameters may be fixed, and the other one may be determined by the charge equality. In particular, for each given Fermi level $\varepsilon_{F}$, one can fix $T$ at the room temperature, $T^{*}=T$, and determine $\mu$ or set $\mu=0$ and calculate the temperature as $T^{*}=c T$. In Table III the values
TABLE III. Values of the density $\rho$, coefficient $c$, and potential $\mu$ for different Fermi levels $\varepsilon_{F}$.

\begin{tabular}{lcccc}
\hline \hline & \multicolumn{4}{c}{$\varepsilon_{F}(\mathrm{eV})$} \\
\cline { 2 - 5 } & 0.3 & 0.4 & 0.5 & 0.6 \\
\hline$\rho\left(\mu \mathrm{m}^{-2}\right)$ & $3.3867 \times 10^{4}$ & $5.9579 \times 10^{4}$ & $9.2638 \times 10^{4}$ & $1.3304 \times 10^{5}$ \\
$c$ & 8.3082 & 11.0197 & 13.7410 & 16.4672 \\
$\mu(\mathrm{eV})$ & 0.1094 & 0.1240 & 0.1354 & 0.1448 \\
\hline \hline
\end{tabular}

of the constant $c$ and the potential $\mu$ for different values of $\varepsilon_{F}$ are reported; for convenience the electron density $\rho$ is also given for each value of $\varepsilon_{F}$.

In the absence of an applied electric field, the mean energy and velocity, calculated with either the Fermi-Dirac or Maxwell-Boltzmann distribution as the initial condition, have to converge to the same stationary values. This convergence is reached for all three Monte Carlo methods, NEMC, SEMC, and FFMC, for different values of the Fermi level $\varepsilon_{F}$. In Fig. 3 the average energy and velocity are shown when the Fermi-Dirac (FD) and Maxwell-Boltzmann (MB) initial conditions are imposed in the FFMC with $\varepsilon_{F}=0.6 \mathrm{eV}$. The same behavior is present when NEMC and SEMC are used (see Figs. 4 and 5).

In the presence of an applied electric field $E$, the mean energy and velocity calculated with the SEMC and NEMC approaches are in good agreement, while they are totally different with the FFMC approach. They are shown in Figs. 6 and 7 , respectively, for $\varepsilon_{F}=0.6 \mathrm{eV}$ and $E=10$ and 20 $\mathrm{kV} / \mathrm{cm}$. The energy given by FFMC with an initial FermiDirac (FFMC-FD) distribution remains about constant, while with an initial Maxwell-Boltzmann distribution (FFMC-MB) it has an initial peak followed by a fast decrease; at $5 \mathrm{ps}$ the values obtained with the FD and MB distributions have a difference of about $9 \%$.

As is evident from Fig. 7, by using an initial MaxwellBoltzmann distribution, the velocity has the same behavior as in Fig. 2(b), with an initial small rising portion followed by a descent toward negative values, which are always higher in absolute value than those obtained by considering the FermiDirac distribution. The difference is considerable, about 50\% for high values of the Fermi energy and the applied electric fields. The choice of an initial high-temperature MaxwellBoltzmann distribution in place of a Fermi-Dirac distribution produces a greater possibility of movement of the particles because there are fewer fully occupied states and the overestimation of the effect of also applying the Pauli principle

(a)
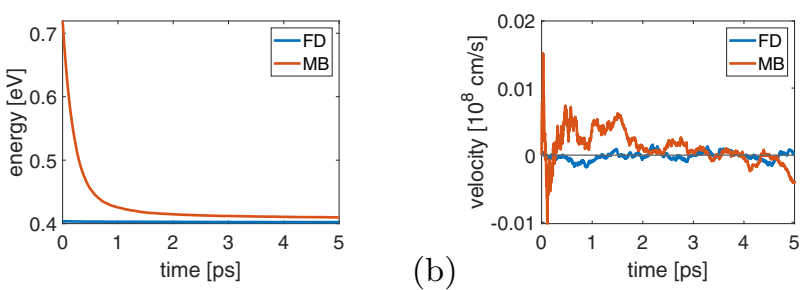

FIG. 3. (a) Mean energy and (b) mean velocity in the FFMC case when $\varepsilon_{F}=0.6 \mathrm{eV}$ and $E=0 \mathrm{kV} / \mathrm{cm}$. 
(a)

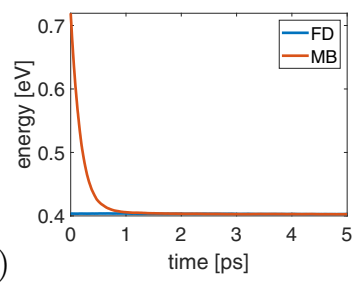

(b)

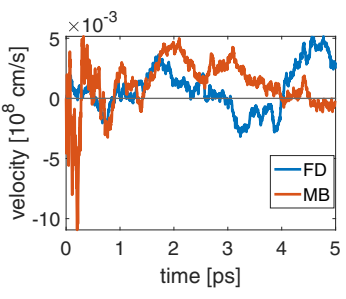

FIG. 4. (a) Mean energy and (b) mean velocity in the NEMC case when $\varepsilon_{F}=0.6$ and $E=0 \mathrm{kV} / \mathrm{cm}$.

at the end of each free flight is smaller, as also noted in [9]. Therefore, the absolute value of the velocity is appreciably higher.

The SEMC and NEMC simulations are not affected by the choice of the initial condition; the mean energy and velocity reach the same stationary values. The only difference is an initial overshoot in the velocity that is not present when an initial Maxwell-Boltzmann distribution is used (see Fig. 8).

\section{B. The charge distribution of the FFMC}

The results obtained using the FFMC procedure, i.e., almost constant mean energies and negative mean velocities, are certainly unphysical and not in agreement with the solution of the Boltzmann equation or with the SEMC and NEMC methods and deserve deeper analysis.

The comparison between the charge distributions obtained in the FFMC simulation when the Fermi-Dirac or the Maxwell-Boltzmann distribution is taken as the initial condition is shown in Fig. 9 for $\varepsilon_{F}=0.6 \mathrm{eV}$ and $E=20 \mathrm{kV} / \mathrm{cm}$. The initial Fermi-Dirac distribution undergoes only negligible changes during the time evolution [see Figs. 9(a) and 9(b)]; the Maxwell-Boltzmann distribution as the initial distribution allows a coarser collocation of the particles with high mean energy because it occupies a portion of the $\mathbf{k}$ space two times larger than that of the initial Fermi-Dirac distribution [see Fig. 9(c)]. The initial MB distribution does not seem to evolve toward a realistic particle distribution, but as time passes, it becomes more similar to an irregular FD-like distribution, as clearly shown in Fig. 9(d). Small voids are present mainly in the front of the distribution due to the effect of the electric field, while the backside is more compact. It is evident that the initial Maxwell-Boltzmann conditions lead to a larger absolute velocity due to the greater possibility of movement of the charged particles. The same considerations hold when the density, i.e., the Fermi level $\varepsilon_{F}$, is lower; in this case the time necessary to reach a FD-like final distribution is longer.
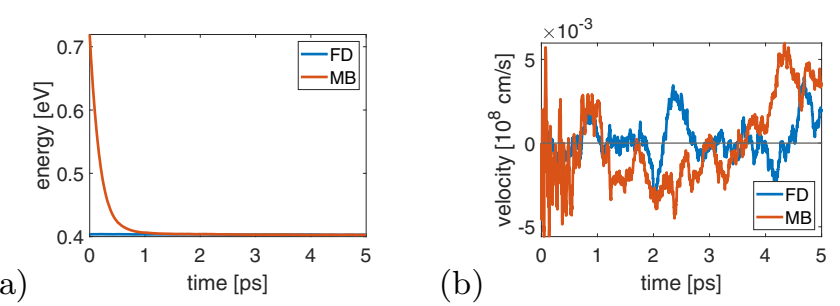

FIG. 5. (a) Mean energy and (b) mean velocity in the EMC case when $\varepsilon_{F}=0.6 \mathrm{eV}$ and $E=0 \mathrm{kV} / \mathrm{cm}$
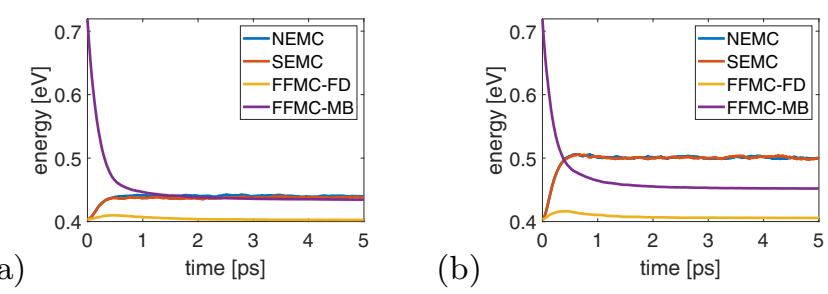

FIG. 6. Mean energy in the NEMC, SEMC, FFMC cases, with FD and $\mathrm{MB}$ initial distributions, when (a) $\varepsilon_{F}=0.6 \mathrm{eV}$ and $E=10$ $\mathrm{kV} / \mathrm{cm}$ and (b) $\varepsilon_{F}=0.6 \mathrm{eV}$ and $E=20 \mathrm{kV} / \mathrm{cm}$.

The dynamics generated by an initial FD distribution is trivial because almost all the final states are occupied during the simulation as a result of the Pauli principle also being imposed at the end of each free flight. When the MB distribution is employed as the initial condition, the dynamics becomes more complex, but the effect seems to be numerical rather than the simulation of a realistic physical situation described by the Boltzmann equations. The results are unphysical; the evolution of the distribution function is not physically satisfactory, and the negative value of the mean velocity has no rationale at all.

\section{Negative mean velocity with the FFMC method}

To understand the origin of the negative mean velocity obtained with the FFMC approach, we investigate the main steps of the Monte Carlo procedure in the three methods, i.e., the free flights and the subsequent scattering events. In the NEMC procedure the distribution function is translated as a whole at each time step $\Delta t$, and the number of the free flights is equal to that of the simulated particles $n_{P}=10^{4}$. In the SEMC scheme, the particles are followed one by one; this leads to an incorrect reconstruction of the distribution function, even if the number of free flights is of the same order as in the NEMC case, as shown in Fig. 10(a) for the case of an initial Fermi-Dirac distribution. In the SEMC and NEMC approaches, the Pauli principle is not imposed at the end of the free flight, only after the scatterings, so that all free flights are allowed and take place. If the Pauli principle is imposed also at the end of the free flights, the number of accepted free flights is strongly reduced, as reported in Fig. 10(b). Also when, initially, the MB distribution is used, the number of free flights is about of 250 at early times but then rapidly decreases and becomes equal to the case with the initial FD distribution. The use of the Pauli principle at the end of each free flight

(a)

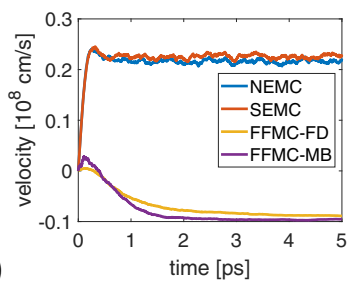

FIG. 7. Mean velocity in the NEMC, SEMC, FFMC cases, with FD and MB initial distributions, when (a) $\varepsilon_{F}=0.6 \mathrm{eV}$ and $E=10$ $\mathrm{kV} / \mathrm{cm}$ and (b) $\varepsilon_{F}=0.6 \mathrm{eV}$ and $E=20 \mathrm{kV} / \mathrm{cm}$. 
(a)

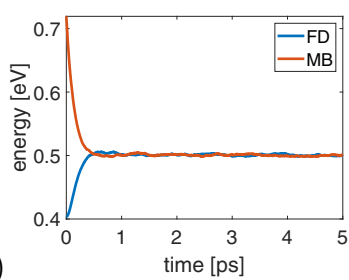

(b)
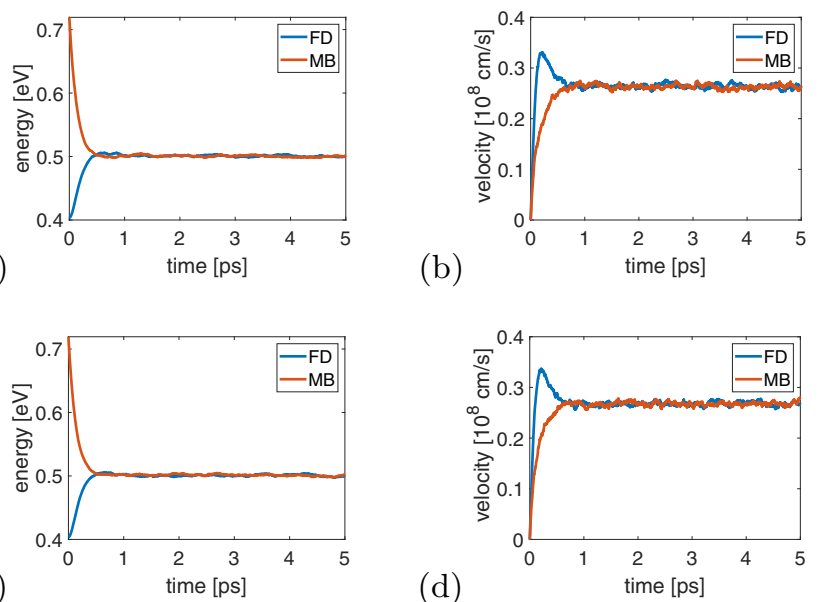

(d)

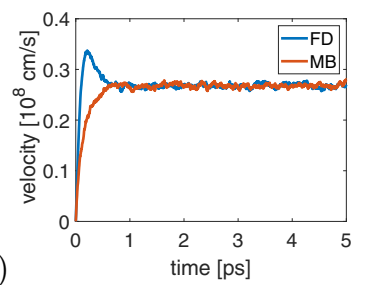

FIG. 8. Mean energy and velocity in the (a) and (b) NEMC and (c) and (d) SEMC cases when the FD or the MB initial distribution is used; $\varepsilon_{F}=0.6 \mathrm{eV}$, and $E=20 \mathrm{kV} / \mathrm{cm}$.

blocks the dynamics; it depletes the numerical sample, and only a few tens of events survive in comparison to the about $10^{4}$ events in the other schemes.

The ratio of the number of accepted free flights with respect to the total is around 0.6 until $t=1.5 \mathrm{ps}$ and drops very much afterward, making the statistics not significant (see Fig. 11).

The free flights, even a few, give a positive contribution to the mean velocity. Therefore, the negative values seen with FFMC in Fig. 7 should originate from the scattering events. The percentage of scatterings whose final states are available is shown in Fig. 12. For the NEMC and SEMC procedures the mean value is about $45 \%$ with a standard deviation of about $4 \%$; in the FFMC case, we observe the same behavior shown in Fig. 11, and after 1.5 ps very few nonrepresentative events are left. In order to understand the negative value of the mean velocity, we analyze all types of scatterings, in particular the fraction of those which lead to a final negative velocity. This fraction is reported in Fig. 13 for the LO and $K$ scatterings (a)

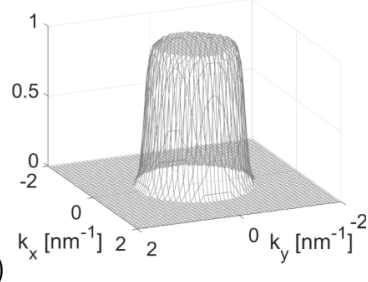

(b)

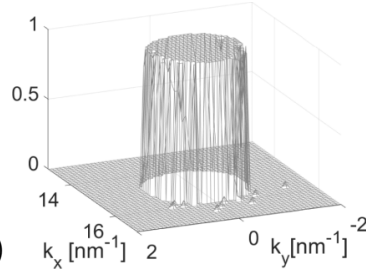

(c)

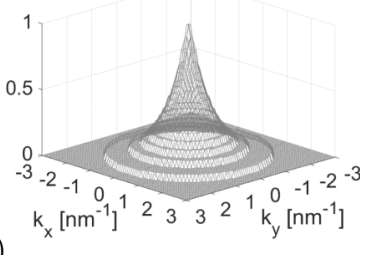

(d)

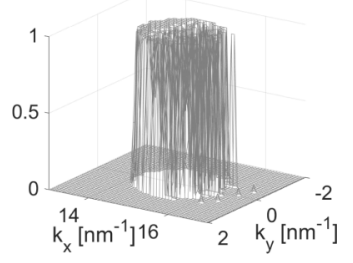

(a)

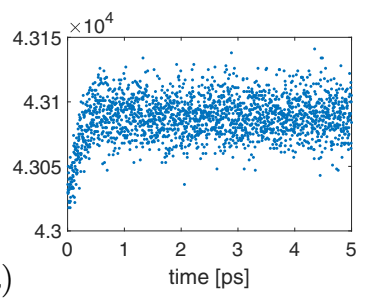

(b)

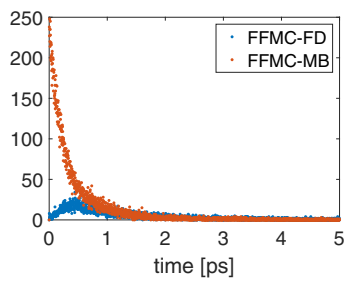

FIG. 10. Number of accepted free flights (a) for SEMC, with the initial FD, and (b) for FFMC when the FD and the MB are used.

in the case of NEMC (the SEMC scheme gives the same qualitative results), while the statistics is again not significant for FFMC. Almost all the $\mathrm{LO}$ and $K$ scatterings produce a negative final velocity in the first $0.5 \mathrm{ps}$; then a mean constant value of about $70 \%$ with a small variance is reached for the LO collisions [see Fig. 13(a)], and a mean constant value of about $80 \%$ is reached for the $K$ scatterings [see Fig. 13(b)].

The previous results show that with FFMC there are very few scattering events; their contribution produces a negative final velocity which cannot be balanced by the free flights because they are almost completely inhibited and are not statistically relevant. This interpretation is confirmed by Figs. 14 and 15. In Fig. 14, the mean velocity is shown when only the scattering events with the LO and $K$ phonons are considered. It is negative in both cases and has a higher absolute value when only the $K$ phonons are included. In Fig. 15 the mean velocity due to only the free flights, without any scattering, is shown. In Fig. 15(a) the initial Fermi-Dirac distribution is used, and the mean value is calculated by considering all the particles of the sample (red line) and only those particles which experience a free flight event at least once (blue line). The two lines are very different, which means that a large number of particles do not experience any free flight during the simulation. The use of the initial MB in place of the FD distribution worsens the results because the mean negative velocity is larger in absolute value with respect to the FD. This is due to the fact that in the early times of the simulation there are more available states and each particle has a free flight event at least once, so that the previous two samples are almost the same, and the corresponding mean velocities are almost equal, as is evident in Fig. 15(b).

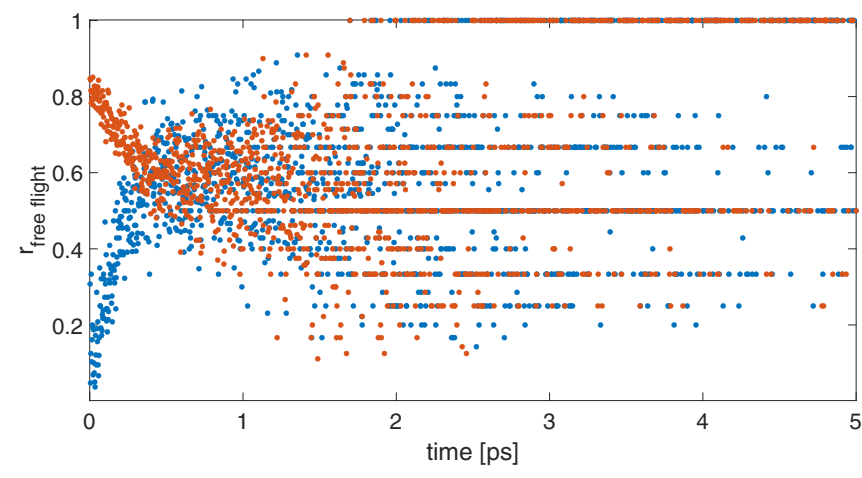

FIG. 11. Ratio of the number of accepted free flights with respect to their total number for the FFMC, with the FD (blue dots) and the MB (red dots) initial conditions. distributions at 5 ps. (c) Initial Initial Fermi-Dirac and (b) charge distributions at 5 ps. $\varepsilon_{F}=0.6 \mathrm{eV}$ and $E=20 \mathrm{kV} / \mathrm{cm}$. 


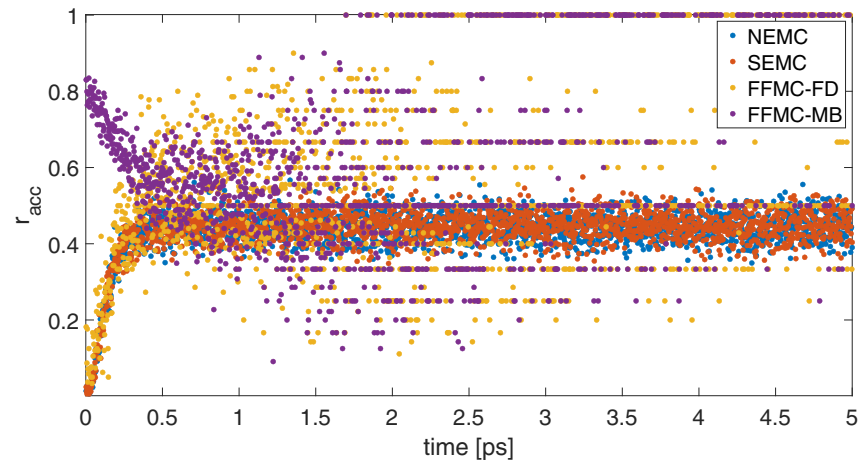

FIG. 12. Ratio of the number of accepted scatterings with respect to their total number for the FFMC scheme, with the FD and MB initial conditions, and the SEMC and NEMC schemes.

When a particle experiences a change in state due to either free flight or scattering with the crystal lattice phonons, it keeps moving in a free motion, without changing its velocity during the rest of the simulation; when this velocity is negative, it will remain negative until the simulation has finished. This fact is supported by the percentage of particles that do not change their velocity between two consecutive time steps, as reported in Fig. 16; after about 1.5 ps, almost all the particles perform only a free motion.

In Fig. 17, the contribution of each type of scattering to the mean velocity, obtained by averaging only over the particle population which experienced the corresponding collision events, is shown. The prevalence of the backward scatterings with LO and $K$ phonons is confirmed; the LA, TA, and TO phonons give a positive contribution, but their number is too low to balance the negative values due to the $\mathrm{LO}$ and $K$ collisions.

\section{CONCLUSIONS}

The inclusion of the Pauli principle in the Monte Carlo simulations by introducing the rejection technique also at the end of each free flight and not only after a scattering event was analyzed by considering a homogeneous suspended monolayer of graphene as a test case. The results were compared with those obtained when the Pauli principle is applied only at the end of the collision events, both in the standard ensemble Monte Carlo and in the updated ensemble Monte Carlo scheme, with the latter being able to take the Pauli principle into account correctly. The treatment of the free
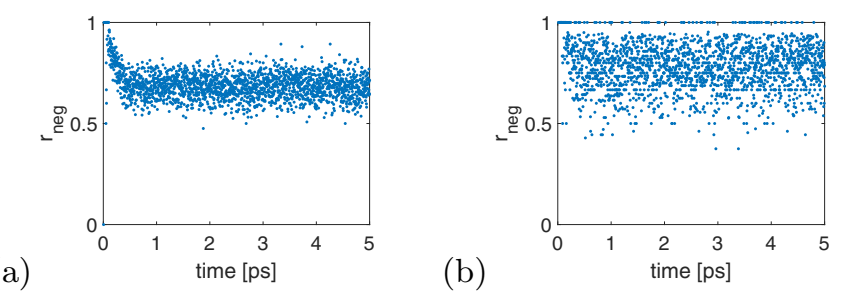

FIG. 13. Ratio of the number of emitted (a) longitudinal-optical and (b) $K$ scatterings, whose final velocity is negative, with respect to their total number for the NEMC approach.

(a)

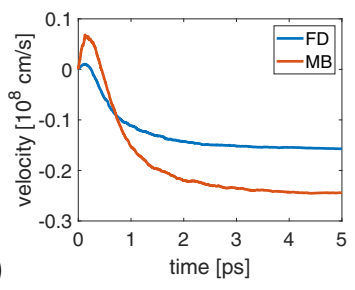

(b)

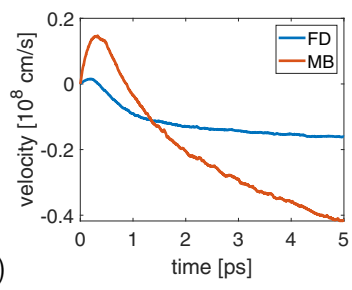

FIG. 14. Mean velocity when (a) only the LO scatterings and (b) only they $K$ scatterings are included for the FFMC, with the FD and the MB initial conditions.

flight in a "quantum" perspective is not in accordance with the Liouvillian nature of the streaming part of the Boltzmann equation. By employing this approach, the resulting numerical dynamics does not seem compatible with the solution of the Boltzmann equations and leads to unphysical results. This is due to the fact that most of the free flights are rejected, the statistics is very poor and becomes nonsignificant, and the distribution function changes very little with respect to the initial one. The mean value of the energy is almost constant, and the mean velocity is negative due to the effect of the backward scatterings, mainly with the longitudinal-optical and $K$ phonons, which are not balanced by the free flights or by the forward scatterings. The same results were also obtained when the initial Fermi-Dirac distribution was substituted by a Maxwell-Boltzmann distribution at high temperature; in this case a greater initial possibility of movement is present, but the charge distribution tends to acquire an irregular FermiDirac-like shape in the course of its evolution. The average values of the velocity are even worse, being negative and larger in absolute value. The average energy starts from a high value and rapidly decreases to a stationary one, in accordance with the evolution of the distribution function. The statistical sample is more populated at early times, but it quickly becomes too poor. For both initial distributions almost all the particles that experience a change in state will not change their velocity during the whole simulation, thus maintaining their state. The FFMC procedure does not reproduce a solution of the Boltzmann equation when degeneracy effects are relevant.

\section{ACKNOWLEDGMENTS}

M.C. acknowledges the research fellowship of the Department of Industrial Engineering and Mathematical Sciences of the Polytechnic University of Marche, "DIPARTIMENTI DI ECCELLENZA -DIISM - Responsabile Prof. Michele

(a)

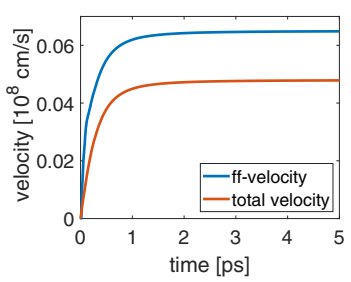

FIG. 15. Total mean velocity and velocity due to the free flight when all the scatterings are neglected for the FFMC with the (a) FD and (b) MB initial conditions. 


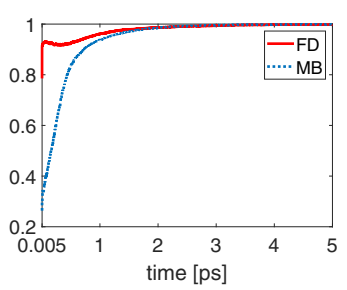

FIG. 16. Percentage of particles whose velocity does not vary at each time step for the FFMC, with the FD and the MB initial conditions.

Germani dal 01.11.2020 (040004-MIUR-DIP-ECCELL2018) CUP I31G18000030005," and "Progetto Giovani 2019" from GNFM (INdAM). P.B., M.C., and V.R. acknowledge the support from GNFM (INdAM).

\section{APPENDIX: THE SCATTERING MECHANISMS}

Here we report the details of the scattering rates for the various phonon branches. For both LA and TA phonons, we consider the elastic approximation, according to which the transition rate is given by [33]

$$
\begin{aligned}
S_{A}\left(\mathbf{k}^{\prime}, \mathbf{k}\right) & =\frac{1}{(2 \pi)^{2}} \frac{\pi D_{a c}^{2} k_{B} T}{2 \hbar \sigma_{m} v_{s}^{2}}\left(1+\cos \vartheta_{\mathbf{k}, \mathbf{k}^{\prime}}\right) \delta\left[\varepsilon\left(\mathbf{k}^{\prime}\right)-\varepsilon(\mathbf{k})\right], \\
s & =\mathrm{LA}, \mathrm{TA},
\end{aligned}
$$

where $D_{a c}$ is the acoustic phonon coupling constant (also called the acoustic phonon deformation potential), $\sigma_{m}$ is the graphene areal density, $v_{s}$ is the sound speed of the sth acoustical phonon branch in graphene, and $\vartheta_{\mathbf{k}, \mathbf{k}^{\prime}}$ is the convex angle between $\mathbf{k}$ and $\mathbf{k}^{\prime}$.

The electron-phonon coupling matrix elements of LO, TO, and $K$ phonons are [20]

$$
\begin{aligned}
& \left|G^{(\mathrm{LO})}\left(\mathbf{k}^{\prime}, \mathbf{k}\right)\right|^{2} \\
& \quad=\frac{1}{(2 \pi)^{2}} \frac{\pi D_{O}^{2}}{\sigma_{m} \omega_{O}}\left[1-\cos \left(\vartheta_{\mathbf{k}, \mathbf{k}^{\prime}-\mathbf{k}}+\vartheta_{\mathbf{k}^{\prime}, \mathbf{k}^{\prime}-\mathbf{k}}\right)\right], \\
& \left|G^{(\mathrm{TO})}\left(\mathbf{k}^{\prime}, \mathbf{k}\right)\right|^{2} \\
& \quad=\frac{1}{(2 \pi)^{2}} \frac{\pi D_{O}^{2}}{\sigma_{m} \omega_{O}}\left[1+\cos \left(\vartheta_{\mathbf{k}, \mathbf{k}^{\prime}-\mathbf{k}}+\vartheta_{\mathbf{k}^{\prime}, \mathbf{k}^{\prime}-\mathbf{k}}\right)\right], \\
& \left|G^{(K)}\left(\mathbf{k}^{\prime}, \mathbf{k}\right)\right|^{2}=\frac{1}{(2 \pi)^{2}} \frac{2 \pi D_{K}^{2}}{\sigma_{m} \omega_{K}}\left(1-\cos \vartheta_{\mathbf{k}, \mathbf{k}^{\prime}}\right),
\end{aligned}
$$

where $D_{O}$ is the optical phonon coupling constant, $\omega_{O}$ is the optical phonon frequency, $D_{K}$ is the $K$ phonon coupling con-

(a)

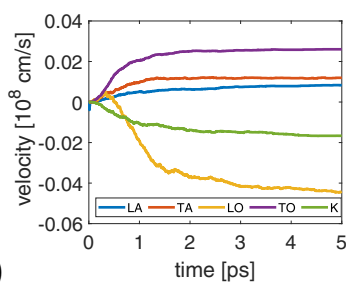

FIG. 17. Contribution of each scattering type to the mean velocity for the FFMC with the (a) FD and (b) MB initial conditions.

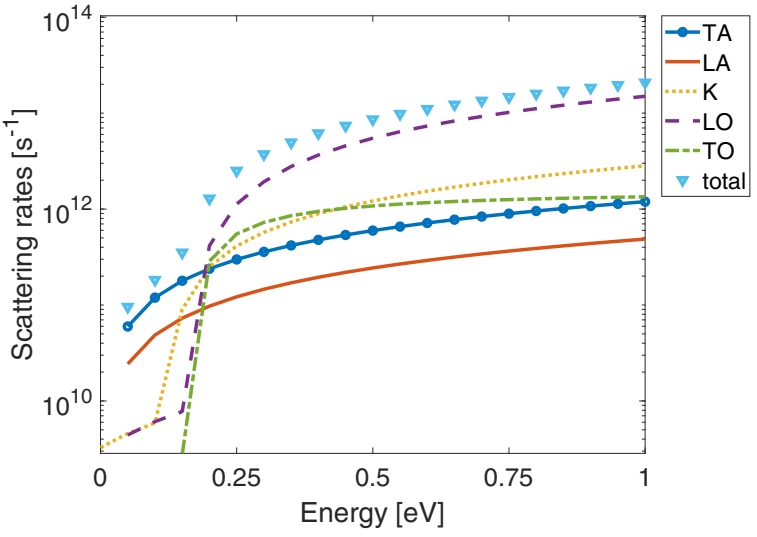

FIG. 18. Scattering rates evaluated with the Bose-Einstein equilibrium distributions for phonons at a temperature of $300 \mathrm{~K}$.

stant, and $\omega_{K}$ is the $K$ phonon frequency. The angles $\vartheta_{\mathbf{k}, \mathbf{k}^{\prime}-\mathbf{k}}$ and $\vartheta_{\mathbf{k}^{\prime}, \mathbf{k}^{\prime}-\mathbf{k}}$ denote the convex angles between $\mathbf{k}$ and $\mathbf{k}^{\prime}-\mathbf{k}$ and between $\mathbf{k}^{\prime}$ and $\mathbf{k}^{\prime}-\mathbf{k}$, respectively.

For the sake of completeness, we provide here the scattering rates of all types of scatterings used in this paper. For the acoustic phonon scattering we get

$$
\Gamma_{\mathrm{ac}}(\varepsilon)=\frac{D_{\mathrm{ac}}^{2} k_{B} T}{4 \hbar^{3} v_{F}^{2} \sigma_{m} v_{s}^{2}} \varepsilon
$$

for the longitudinal- and transverse-optical phonons we get

$$
\begin{aligned}
\Gamma_{\mathrm{LO}, \mathrm{TO}}(\varepsilon)= & \frac{D_{O}^{2}}{4 \pi \sigma_{m} \omega_{O} \hbar^{2} v_{F}^{2}}\left\{\left(\varepsilon-\hbar \omega_{O}\right)\left(g_{\mathrm{LO}, \mathrm{TO}}+1\right)\right. \\
& \times H\left(\varepsilon-\hbar \omega_{O}\right)\left[2 \pi \mp \Lambda^{-}(\varepsilon)\right] \\
& \left.+\left(\varepsilon+\hbar \omega_{O}\right) g_{\mathrm{LO}, \mathrm{TO}}\left[2 \pi \mp \Lambda^{+}(\varepsilon)\right]\right\}, \quad
\end{aligned}
$$

where the upper and lower signs refer to the LO and TO phonons, respectively. $H$ is the Heaviside function, and

$$
\begin{aligned}
& \Lambda^{ \pm}(\varepsilon) \\
& =\int_{0}^{2 \pi} \frac{\left(2 \varepsilon^{2}+\hbar^{2} \omega_{O}^{2} \pm 2 \hbar \omega_{O} \varepsilon\right) \cos \theta^{\prime \prime}-2 \varepsilon\left(\varepsilon \pm \hbar \omega_{O}\right)}{2 \varepsilon^{2}+\hbar^{2} \omega_{O}^{2} \pm 2 \hbar \omega_{O} \varepsilon-2 \varepsilon\left(\varepsilon \pm \hbar \omega_{O}\right) \cos \theta^{\prime \prime}} d \theta^{\prime \prime} \\
& =-\frac{\pi}{\varepsilon\left(\varepsilon \pm \hbar \omega_{O}\right)}\left(2 \varepsilon^{2}+\hbar^{2} \omega_{O}^{2} \pm 2 \hbar \omega_{O} \varepsilon-\hbar \omega_{O}\left|2 \varepsilon \pm \hbar \omega_{O}\right|\right) .
\end{aligned}
$$

Similarly, the expression for the scattering rate for the $K$ phonon scattering reads

$$
\begin{aligned}
\Gamma_{K}(\varepsilon)= & \frac{D_{K}^{2}}{\sigma_{m} \omega_{K} \hbar^{2} v_{F}^{2}}\left[\left(\varepsilon-\hbar \omega_{K}\right)\left(g_{K}+1\right)\right. \\
& \left.\times H\left(\varepsilon-\hbar \omega_{K}\right)+\left(\varepsilon+\hbar \omega_{K}\right) g_{K}\right] .
\end{aligned}
$$

These scattering rates are shown in Fig. 18 as a function of the energy. 
[1] P. A. Lebwohl and P. J. Price, Hybrid method for hot electron calculations, Solid State Commun. 9, 1221 (1971).

[2] S. Bosi and C. Jacoboni, Monte Carlo high-field transport in degenerate GaAs, J. Phys. C 9, 315 (1976).

[3] C. Jacoboni and L. Reggiani, The Monte Carlo method for the solution of charge transport in semiconductors with applications to covalent material, Rev. Mod. Phys. 55, 645 (1983).

[4] P. Lugli and D. K. Ferry, Degeneracy in the ensemble Monte Carlo method for high-field transport in semiconductors, IEEE Trans. Electron Devices 32, 2431 (1985).

[5] P. Borowik and J. L. Thobel, Improve Monte Carlo method for the study of electron transport in degenerate semiconductors, J. Appl. Phys. 84, 3706 (1998).

[6] P. Borowik and L. Adamowciz, Improved algorithm for Monte Carlo studies of electron transport in degenerate semiconductors, Phys. B (Amsterdam, Neth.) 365, 235 (2005).

[7] M. V. Fischetti and S. E. Laux, Monte Carlo analysis of electron transport in small semiconductor devices including band-structure and space-charge effects, Phys. Rev. B 38, 9721 (1988).

[8] M. Zebarjadi, C. Bulutay, K. Esfarjani, and A. Shakouri, Monte Carlo simulation of electron transport in degenerate and inhomogeneous semiconductors, Appl. Phys. Lett. 90, 092111 (2007).

[9] P. Tadyszak, F. Danneville, A. Cappy, L. Reggiani, L. Varani, and L. Rota, Monte Carlo calculations of hot-carrier noise under degenerate conditions, Appl. Phys. Lett. 69, 1450 (1996).

[10] D. K. Ferry and S. M. Goodnick, Ensemble Monte Carlo simulations of ultrafast phenomena in semiconductors, in Ultrafast Phenomena in Semiconductors, edited by K. T. Tsen (Springer, New York, 2001), pp. 307-352.

[11] A. Islam and K. Kalna, Monte Carlo simulations of mobility in doped GaAs using self-consistent Fermi-Dirac statistics, Semicond. Sci. Technol. 26, 055007 (2011).

[12] V. Romano, A. Majorana, and M. Coco, DSMC method consistent with the Pauli exclusion principle and comparison with deterministic solutions for charge transport in graphene, J. Comput. Phys. 302, 267 (2015).

[13] M. Coco, A. Majorana, and V. Romano, Cross validation of discontinuous Galerkin method and Monte Carlo simulations of charge transport in graphene on substrate, Ric. Mat. 66, 201 (2017).

[14] M. Coco, A. Majorana, G. Nastasi, and V. Romano, High-field mobility in graphene on substrate with a proper inclusion of the Pauli exclusion principle, Atti Accad. Peloritana Pericolanti, Cl. Sci. Fis., Mat. Nat. 97(suppl. 1), A6 (2019).

[15] M. Coco, A. Majorana, G. Mascali, and V. Romano, Comparing kinetic and hydrodynamical models for electron transport in monolayer graphene, in Coupled Problems 2015: Proceedings of the 6th International Conference on Coupled Problems in Science and Engineering, edited by B. Schrefler, E. Oñate and M. Papadrakakis (CIMNE, Venice, 2015), p. 1003.
[16] G. Nastasi and V. Romano, Improved mobility models for charge transport in graphene, Commun. Appl. Ind. Math. 10, 41 (2019).

[17] G. Nastasi and V. Romano, A full coupled drift-diffusionPoisson simulation of a GFET, Commun. Nonlinear Sci. Numer. Simulat. 87, 105300 (2020).

[18] M. Coco and G. Nastasi, Simulation of bipolar charge transport in graphene on h-BN, COMPEL Int. J. Comput. Math. Electr. Electron. Eng. 39, 449 (2020).

[19] A. Majorana, G. Nastasi, and V. Romano, Simulation of bipolar charge transport in graphene by using a discontinuous Galerkin method, Commun. Comput. Phys. 26, 114 (2019).

[20] P. Lichtenberger, O. Morandi, and F. Schürrer, High-field transport and optical phonon scattering in graphene, Phys. Rev. B 84 045406 (2011).

[21] G. A. Bird, Direct simulation and the Boltzmann equation, Phys. Fluids 13, 2676 (1970).

[22] G. A. Bird, Molecular Gas Dynamics (Clarendon, Oxford, 1976).

[23] W. Wagner, A convergence proof for Bird's direct simulation Monte Carlo method for the Boltzmann equation, J. Stat. Phys. 66, 1011 (1992).

[24] C. Jacoboni, Theory of Electron Transport in Semiconductors (Springer, Berlin, 2010).

[25] A. H. Castro Neto, F. Guinea, N. M. R. Peres, K. S. Novoselov, and A. K. Geim, The electronic properties of graphene, Rev. Mod. Phys. 81, 109 (2009).

[26] M. Coco and V. Romano, Simulation of electron-phonon coupling and heating dynamics in suspended monolayer graphene including all the phonon branches, J. Heat Transfer 140, 092404 (2018).

[27] G. Mascali and V. Romano, Charge transport in graphene including thermal effects, SIAM J. Appl. Math. 77, 593 (2017).

[28] M. Coco, G. Mascali, and V. Romano, Monte Carlo analysis of thermal effects in monolayer graphene, J. Comput. Theor. Transport 45, 540 (2016).

[29] M. Coco and V. Romano, Assessment of the constant phonon relaxation time approximation in electron-phonon coupling in graphene, J. Comput. Theor. Transport 47, 246 (2018).

[30] C. Jacoboni and P. Lugli, The Monte Carlo Method for Semiconductor Device Simulation (Springer, Vienna 1989).

[31] X. Li, E. A. Barry, J. M. Zavada, M. Buongiorno Nardelli, and K. W. Kim, Surface polar phonon dominated electron transport in graphene, Appl. Phys. Lett. 97, 232105 (2010).

[32] K. M. Borysenko, J. T. Mullen, E. A. Barry, S. Paul, Y. G. Semenov, J. M. Zavada, M. Buongiorno Nardelli, and K. W. Kim, First-principles analysis of electron-phonon interactions in graphene, Phys. Rev. B 81, 121412(R) (2010).

[33] S. Das Sarma, S. Adam, E. H. Hwang, and E. Rossi, Electronic transport in two-dimensional graphene, Rev. Mod. Phys. 83, 407 (2011). 